\title{
Biologically active 1,25-dihydroxyvitamin D3 protects against experimental sepsis by negatively regulating the Toll-like receptor $4 /$ myeloid differentiation primary response gene 88/Toll-IL-1 resistance-domain-containing adapter-inducing interferon- $\beta$ signaling pathway
}

\author{
GE ZHENG, NA WEN, MINLI PAN, YUMAO HUANG and ZHISHU LI \\ Department of Pediatrics, Ruian People's Hospital, Ruian, Zhejiang 325200, P.R. China
}

Received November 3, 2018; Accepted June 18, 2019

DOI: $10.3892 /$ ijmm.2019.4266

\begin{abstract}
The hormonally active form of vitamin D (VD), 1,25-dihydroxyvitamin $\mathrm{D} 3$, has been reported to be a key immunoregulator in the reduction of inflammation. In this study, we investigated the effects of VD in an experimental sepsis cell model, and the underlying mechanisms. The sepsis cell model was first established in monocytes, isolated from newborns and healthy adults, which were stimulation with lipopolysaccharide (LPS). We observed that cell viability was significantly impaired in the monocytes after LPS stimulation, using a Cell Counting Kit-8 and trypan blue assays. Additionally, ELISA revealed that LPS stimulation significantly elevated the expression of interleukin 6 (IL-6), IL-10 and tumor necrosis factor- $\alpha$ (TNF- $\alpha$ ). The expression levels of Toll-like receptor (TLR4), myeloid differentiation primary response gene 88 (MyD88), and Toll-IL-1 resistance-domain-containing adapter-inducing interferon- $\beta$ (TRIF) mRNA were also significantly elevated under LPS stimulation using reverse transcription-quantitative PCR and western blot analysis. VD treatment could significantly suppress the effects of LPS simulation on monocytes by negatively regulating inflammatory cytokines and TLR4/MyD88/TRIF signaling. Furthermore, a regulatory feedback mechanism was proposed to involve TLR4, MyD88 and TRIF in the sepsis cell model. In conclusion, VD may effectively decrease the release of inflammatory cytokines by inhibiting the TLR4/MyD88/TRIF signaling pathway, could be considered as a potential therapeutic agent for the treatment of sepsis.
\end{abstract}

Correspondence to: Dr Zhishu Li, Department of Pediatrics, Ruian People's Hospital, 108 Wangsong Road, Ruian, Zhejiang 325200, P.R. China

E-mail:66209875@qq.com

Key words: 1,25-dihydroxyvitamin D3, sepsis, monocytes, Toll-like receptor 4/myeloid differentiation primary response gene 88/Toll-IL-1 resistance-domain-containing adapter-inducing interferon- $\beta$ signaling

\section{Introduction}

Sepsis is a life-threatening clinical syndrome, and is characterized by systemic inflammatory reactions that occur due to infection (1). It is a serious complication of severe trauma, burns and surgery, and is also a major cause of infections and multiple organ dysfunction syndrome (2-4). Sepsis arises as a result of the complex interactions of pro- and anti-inflammatory processes caused by factors, such as Gram-negative and Gram-positive bacteria; the competition between the host immune response and pathogens determines patient survival (5). In the United States of America, the morbidity from severe sepsis has been reported to be $\sim 300$ cases per 100,000 residents, with a $25 \%$ fatality rate (6). The mortality of patients is the highest in those with respiratory tract infections, particularly pneumonia (6). At present, the administration of immunostimulatory therapy to patients at the appropriate stage represents a major advancement in sepsis treatment.

The majority of patients who are critically ill are deficient in vitamin D [serum 1,25-dihydroxyvitamin D3 (VD) concentrations <20-30 ng/ml] (7). Patients with sepsis in emergency departments and intensive care units generally exhibit lower levels of serum VD compared with healthy controls $(8,9)$. A previous study revealed a correlation between VD deficiency and high mortality in sepsis patients (10). Besides regulating calcium-phosphate homeostasis, VD affects innate and adaptive immunity and their interplay; VD may induce immune imbalance, potentially affecting the pathogenesis of certain infections $(11,12)$. Upregulated expression of biomarkers associated with sepsis-induced systemic inflammation are important indicators of VD deficiency (9); however, few studies have investigated the specific mechanisms by which VD modulates the inflammatory response in patients with sepsis.

Toll-like receptor (TLR) family members (TLR1-TLR10) serve a critical role in mediating immune responses against microbial infections via the recognition of microbial components, including bacterial lipopolysaccharide, lipoproteins, peptidoglycan and single-stranded and double-stranded viral RNAs $(13,14)$. Induction of TLR signaling pathways arises 
from the conserved cytoplasmic Toll/interleukin (IL)-1 receptor (TIR) domains of vertebrate TLRs (15). The myeloid differentiation primary response gene 88 (MyD88) contains the TIR domain at the C-terminal. MyD88 was identified as an important adapter for all TLRs, recruiting IL-1 receptor-associated kinase to TLRs, activating downstream effector proteins to stimulate the secretion of inflammatory cytokines $(16,17)$. Two other TIR domain-containing molecules, TIR domain-containing Adapter Protein (TIRAP) and TIR-domain-containing adapter-inducing interferon- $\beta$ (TRIF), have been identified to be involved in the TLR-signaling pathways (18). Specifically, TIRAP participates in the TLR2- and TLR4-MyD88-dependent signaling pathway, whereas the adapter TRIF has a critical role in TLR3- and TLR4-mediated MyD88-dependent signaling $(19,20)$.

Monocytes serve a critical role in mediating anti-bacterial responses (21), and an abnormal inflammatory response of monocytes could result in severe pathologies, such as sepsis (22). In sepsis, monocytes recognize Gram-negative bacteria (endotoxins) and induce a dysfunctional immuneinflammatory response via TLR4 $(23,24)$, in which MyD88and TRIF-dependent pathways are involved (25). The expression of pro-inflammatory cytokine genes, including IL-6, C-X-C motif ligand (CXCL)1, chemokine ligand (CCL)3, and tumor necrosis factor (TNF) via activation of nuclear factor- $\mathrm{\kappa B}(26,27)$ can be triggered via the MyD88-dependent pathway (28). On the contrary, the expression of type I IFNs (IFN- $\alpha$ and IFN- $\beta$ ) and IFN-inducible chemokines, such as CXCL10, CCL5 and CCL2 (19) can be induced by the TRIF-dependent pathway. TRIF also regulates the differentiation of monocytes to dendritic cells and their mobilization to the lymph nodes (29). A previous study reported that VD regulates the TLR4 pathway in colon cancer, induced by inflammatory stimuli (30). Sadeghi et al (31) demonstrated a dose-dependent decrease in TLR2 and TLR4 mRNA and protein expression in VD-treated human monocytes. We hypothesized a strong association between VD and TLR signaling, particularly via TLR4, in sepsis.

In the present study, cell viability, inflammatory cytokine production, and the expression of TLR4-signaling components were determined in a lipopolysaccharide (LPS)-induced sepsis model using human newborn and adult monocytes. We further investigated whether VD has an effect on the sepsis-induced inflammation response through the TLR4 signaling pathway. Our findings may improve understanding of the action of $\mathrm{VD}$ in anti-inflammatory responses in bacterial pathogen infections.

\section{Materials and methods}

Monocytes isolation from newborns and healthy adults. Peripheral blood was collected from 20 healthy newborns (12 male and 8 female) and 20 healthy adult volunteers (10 male and 10 female, 46.7 \pm 21.3 years old) from March 2017 to January 2018 according to a protocol approved by the Ethics Committee of the Ruian People's Hospital. Prior to peripheral blood collection, written informed consent was provided by the pregnant females and volunteers. None of the subjects had a history of intra-amniotic infection or any other infection. Blood collected from left arm underwent anti-coagulation by the addition of heparin $(10 \mathrm{U} / \mathrm{ml})$. Monocytes in the form of peripheral blood mononuclear cells (PBMCs) were extracted from peripheral blood, using Ficoll Hypaque (GE Healthcare) density gradient centrifugation according to the manufacturer's protocols. The isolated cells were cultured in RPMI-1640 medium (HyClone; GE Healthcare Life Sciences) supplemented with $10 \%$ fetal bovine serum (Gibco; Thermo Fisher Scientific, Inc.) and $1 \%$ penicillin/streptomycin (Beyotime Institute of Biotechnology). Subsequently, the cells were maintained in 24-well plates at $37^{\circ} \mathrm{C}$.

Establishment of a sepsis cell model. LPS from E. coli, Serotype R 1515 (Re; liquid) was purchased from Alexis Biochemicals and used at a concentration of $10 \mathrm{ng} / \mathrm{ml}$. For the induction of the sepsis cell model, PBMCs $\left(5 \times 10^{6}\right.$ cells $\left./ \mathrm{ml}\right)$ were seeded onto 6-well plates and stimulated without or with LPS of different concentrations (10, 20, 40 and $80 \mathrm{ng} / \mathrm{ml})$ at indicated time points $(24,48$ and $72 \mathrm{~h}), 37^{\circ} \mathrm{C}$, and were classified into four groups: Newborn control (NC), newborn sepsis (NS), adult control (AC) and adult sepsis (AS). PBS was used as a control. The cell viability assay on diverse of concentrations of LPS was performed to determine the cytotoxic effects.

Treatment of monocytes with VD. VD (Nature's Bounty) was dissolved and diluted in RPMI-1640 medium to final concentrations of $10^{-7}, 10^{-9}$ and $10^{-11} \mathrm{M}$. Then, the monocytes were pre-treated with or without VD $\left(10^{-7}, 10^{-9}\right.$ or $\left.10^{-11} \mathrm{M}\right)$ for $4 \mathrm{~h}$, stimulated with or without LPS $(10 \mathrm{ng} / \mathrm{ml})$ for $48 \mathrm{~h}$ at $37^{\circ} \mathrm{C}$, and incubated in a humidified $5 \% \mathrm{CO}_{2}$. environment at $37^{\circ} \mathrm{C}$.

Suppressing TLR4 expression in monocytes. Monocytes from the four groups (NC, NS, AC and AS) were pre-incubated with $1 \mu \mathrm{g} / \mathrm{ml}$ anti-TLR4 (ab22048) or IgG2b (ab91366, both Abcam) for $1 \mathrm{~h}$ and stimulated with VD $\left(10^{-9} \mathrm{M}\right)$ for $48 \mathrm{~h}$ at $37^{\circ} \mathrm{C}$ to give a total of 16 groups, which were as follows: $\mathrm{NC}+\mathrm{IgG}$, $\mathrm{NC}+\mathrm{TLR} 4, \mathrm{NS}+\mathrm{IgG}, \mathrm{NS}+\mathrm{TLR} 4, \mathrm{AC}+\mathrm{IgG}, \mathrm{AC}+\mathrm{TLR} 4$, $\mathrm{AS}+\mathrm{IgG}, \mathrm{AS}+\mathrm{TLR} 4, \mathrm{NC}+\mathrm{IgG}+\mathrm{VD}, \mathrm{NC}+\mathrm{TLR} 4+\mathrm{VD}$, $\mathrm{NS}+\mathrm{IgG}+\mathrm{VD}, \mathrm{NS}+\mathrm{TLR} 4+\mathrm{VD}, \mathrm{AC}+\mathrm{IgG}+\mathrm{VD}$, $\mathrm{AC}+\mathrm{TLR} 4+\mathrm{VD}, \mathrm{AS}+\mathrm{IgG}+\mathrm{VD}$ and AS + TLR4 + VD. Comparisons were performed between the groups with TLR4 and without TLR4.

Plasmid transfection. Small interfering RNA (si)MyD88 and siTRIF, and a negative control (siCon) were chemically synthesized by Shanghai GenePharma Co., Ltd. (Shanghai, China). The sequences were as follows: siNC, 5'-GCGACG AUCUGCCUAAGAUTT-3'; siMyD88, 5'-GCCUGUCUC UGUUCUUGAATT-3' and SiTRIF, 5'-GCCAGCAACUUG GAAAUCATT-3'. To determine the function of MyD88 and TRIF in vitro, monocytes from the four groups (NC, NS, AC, and AS) were transfected with $10 \mathrm{nM}$ siMyD88, siTRIF and siCon using Lipofectamine ${ }^{\circledR} 2000$ (Invitrogen; Thermo Fisher Scientific, Inc.) according to the manufacturer's instructions and stimulated with VD $\left(10^{-9} \mathrm{M}\right)$ for $48 \mathrm{~h}$ at $37^{\circ} \mathrm{C}$. For MyD88 knockdown, the monocytes were further classified into 16 groups as follows: $\mathrm{NC}+$ siCon, $\mathrm{NC}+$ siMyD88, $\mathrm{NS}+$ siCon, $\mathrm{NS}+$ siMyD88, AC + siCon, AC + siMyD88, AS + siCon, $\mathrm{AS}+$ siMyD88, NC + siCon + VD, NC + siMyD88 + VD, $\mathrm{NS}+$ siCon + VD, NS + siMyD88 + VD, AC + siCon + VD, $\mathrm{AC}+\mathrm{siMyD} 88+\mathrm{VD}, \mathrm{AS}+$ siCon+ VD and AS + siMyD88 + VD. 
Correspondingly, a total of 16 groups, including $\mathrm{NC}+$ siCon, $\mathrm{NC}+$ siTRIF, NS + siCon, NS + siTRIF, AC + siCon, $\mathrm{AC}+$ siTRIF, AS + siCon, AS + siTRIF, NC + siCon + VD, $\mathrm{NC}+$ siTRIF + VD, NS + siCon + VD, NS + siTRIF + VD, $\mathrm{AC}+$ siCon + VD, AC + siTRIF + VD, AS + siCon + VD and $\mathrm{AS}+\mathrm{siTRIF}+\mathrm{VD}$ were used to investigate the function of TRIF in the sepsis cell model. Comparisons were performed between the groups transfected with siCon and siMyD88 or siTRIF.

Cell Counting Kit-8 (CCK-8) assay. Three independent experiments were performed to assess the cytotoxic effects of LPS on monocytes from the AC and NC groups using a CCK- 8 assay (Dojindo Molecular Technologies, Inc.) according to the manufacturer's protocols. Briefly, the monocytes were seeded onto a 96-well plate at a density of 3,000 cells per well and incubated for $24 \mathrm{~h}$ at $37^{\circ} \mathrm{C}$. The monocytes were then treated as indicated. After incubation with 24,48 and $72 \mathrm{~h}$, the CCK-8 reagent $(10 \mu \mathrm{l})$ was added to each well and $4 \mathrm{~h}$ later, each sample was measured at $450 \mathrm{~nm}$ using a BioTek microplate reader.

Trypan blue assay. The viability of the different monocyte groups was determined by trypan blue staining. Briefly, the monocytes were seeded onto each well of the 24-well culture dish. When they reached $60-70 \%$ confluence, trypsin and an equal volume of trypan blue $(0.2 \%)$ were added. The cell viability was observed after incubation for 1-2 $\mathrm{min}$ at room temperature with trypan blue using light microscopy. There are four 1 x $1 \mathrm{~mm}$ squares of one chamber and the average number of cells per square (all hemocytometers consist of two chambers, each is divided into nine $1 \mathrm{~mm}^{2}$ squares) is determined. For an accurate determination, cells in 4 corner squares plus the central big square (20-50 cells/square) were analyzed. If the cell density is higher than 200 cells/square, cell suspension should be further diluted.

Cytokine analysis. To confirm effective cell stimulation by the various treatments, the levels of key pro-inflammatory cytokines, TNF- $\alpha$ and IL-6, and the anti-inflammatory cytokine IL-10, were determined in the supernatants from monocytes using ELISA. Briefly, the levels of IL-6, IL-10, and TNF- $\alpha$ in the cell culture supernatants collected after 24,48 and $72 \mathrm{~h}$ incubation were determined using commercial ELISA kits (cat. nos. GR106328-2, GR106292-2 and GR106397-2, respectively, Genorise Scientific Inc.) according to the manufacturer's instructions.

Reverse transcription-quantitative PCR (RT-qPCR). Monocytes were collected, and cellular RNAs from the different cell groups were extracted using TRIzol ${ }^{\circledR}$ reagent (Thermo Fisher Scientific, Inc.) and then reverse-transcribed into cDNA using PrimeScript ${ }^{\mathrm{TM}}$ RT reagent Kit with gDNA Eraser (Takara Bio, Inc.) according to manufacturer's instructions. qPCR was performed using an ABI 7300 RT-PCR system (Applied Biosystems; Thermo Fisher Scientific, Inc.). After determining RNA concentration, in vitro RT was performed in a $20 \mu \mathrm{l}$ with a system including $4 \mu 125 \mathrm{mM}$ $\mathrm{MgCl}_{2}, 2 \mu \mathrm{l}$ Reverse Transcription 10X Buffer, $2 \mu 110 \mathrm{mM}$ dNTP Mixture, $0.5 \mu$ l Ribonuclease Inhibitor, $15 \mathrm{U}$ AMV Reverse Transcriptase, $0.5 \mu \mathrm{g}$ random Primers, $1 \mu \mathrm{g}$ total RNA and Nuclease-Free Water to a final volume of $20 \mu 1$ under $42^{\circ} \mathrm{C}$ for $15 \mathrm{~min}$ and $85^{\circ} \mathrm{C}$ denaturation (Promega Corporation). qPCR was then performed using SYBR Premix Ex Taq GC kit (Takara Bio, Inc.) with $7.5 \mu \mathrm{l} 2 \mathrm{X}$ premix, $10 \mathrm{mM}$ forward and reverse primers and $\mathrm{dH}_{2} \mathrm{O}$ to a final volume of $15 \mu \mathrm{l}$ under the following conditions: $94^{\circ} \mathrm{C}$ denaturation for $30 \mathrm{sec}$, followed by 40 cycles each containing $94^{\circ} \mathrm{C}$ denaturation for $5 \mathrm{sec}$, and $60^{\circ} \mathrm{C}$ annealing for $30 \mathrm{sec}$, final extension of $72^{\circ} \mathrm{C}$ for $2 \mathrm{~min}$ with a LightCycler 480 instrument (Roche Diagnostics). The primer sequences were used as follows: VDR, forward 5'-CCC TTGACCTCTTCCCGCTGGTT-3', reverse, 5'-TCACTG ACGCGGTACTTGTAGTCTTGGTTG-3'; TLR4, forward 5'-AGACCTGTCCCTGAACCCTAT-3', reverse, 5'-CGA TGGACTTCTAAACCAGCCA-3'; MyD88, forward 5'-GGC TGCTCTCAACATGCGA-3', reverse, 5'-CTGTGTCCG CACGTTCAAGA-3'; TRIF, forward 5'-CCTGGAATCATC ATCGGAACAG-3', reverse 5'-TGAGTGGTCTATGGCGT CCT-3'; and GAPDH, forward 5'-TGTTCGTCATGGGTG TGAAC-3', reverse, 5'-ATGGCATGGACTGTGGTCAT-3'. All the samples were analyzed in triplicates, and the $2^{-\Delta \Delta C q}$ method was used to calculate the gene expression levels (32).

Western blot analysis. For western blot analysis, protein (30-50 $\mu \mathrm{g})$ extracts were obtained by lysing the monocytes in a buffer as previously reported (33). After protein quantitation using a BCA protein assay, protein extracts were separated by $10 \%$ SDS-PAGE and transferred onto a polyvinylidene difluoride membrane. The membrane was blocked with $10 \%$ skim milk at room temperature for $1 \mathrm{~h}$, probed with primary antibodies against TLR4 (sc-293072, 1:1,000), MyD88 (sc-74532, 1:1,000), and TRIF (sc-514384, 1:1,000) from Santa Cruz Biotechnology, Inc., and GAPDH (cat. no. 14C10, 1:1,000, Cell Signaling Technology, Inc.) overnight at $4^{\circ} \mathrm{C}$. Then, the membrane was incubated with a horseradish peroxidase-conjugated secondary antibody (PI31430, 1:10,000; Pierce; Thermo Fisher Scientific, Inc.) at room temperature for $1 \mathrm{~h}$. The signals were visualized using enhanced chemiluminescence detection (GE Healthcare). Band densities were quantified with ImageJ $1.43 \mathrm{r}$ (National Institutes of Health).

Statistical analysis. Statistical analysis was conducted using GraphPad Prism software version 6 (GraphPad Software, Inc.). The results are expressed as the mean \pm standard deviation of at least three independent experiments. The differences between groups were assessed using one-way analysis for variance followed by a Tukey's post-hoc test. $\mathrm{P}<0.05$ was considered to indicate a statistically significant difference.

\section{Results}

Establishment and evaluation of the sepsis cell model. To construct a sepsis cell model, the monocytes from newborns and healthy adults were stimulated with LPS or control (PBS) for 24,48 or $72 \mathrm{~h}$. After treatment, no significant difference in the expression of VD receptor (VDR) mRNA expression was detected among the groups (Fig. 1A). Subsequently, different concentrations of LPS 10, 20, 40 and $80 \mathrm{ng} / \mathrm{ml}$ were tested. The cell viability assay indicated that 40 and $80 \mathrm{ng} / \mathrm{ml}$ LPS exhibited significant cytotoxic effects in the NS and AS groups compared with the respective controls (Fig. 1B). We thus used 


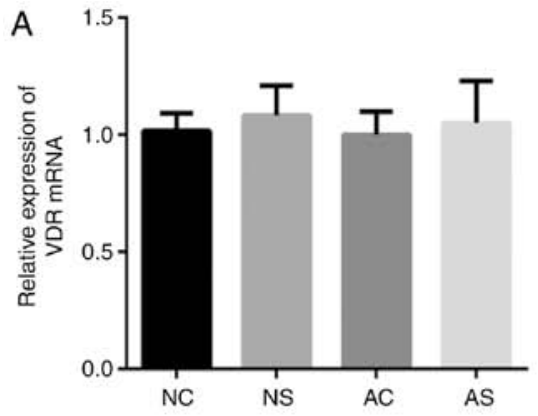

C

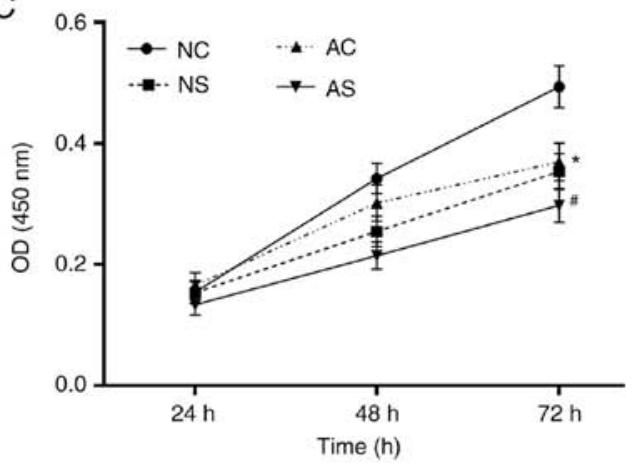

$E$
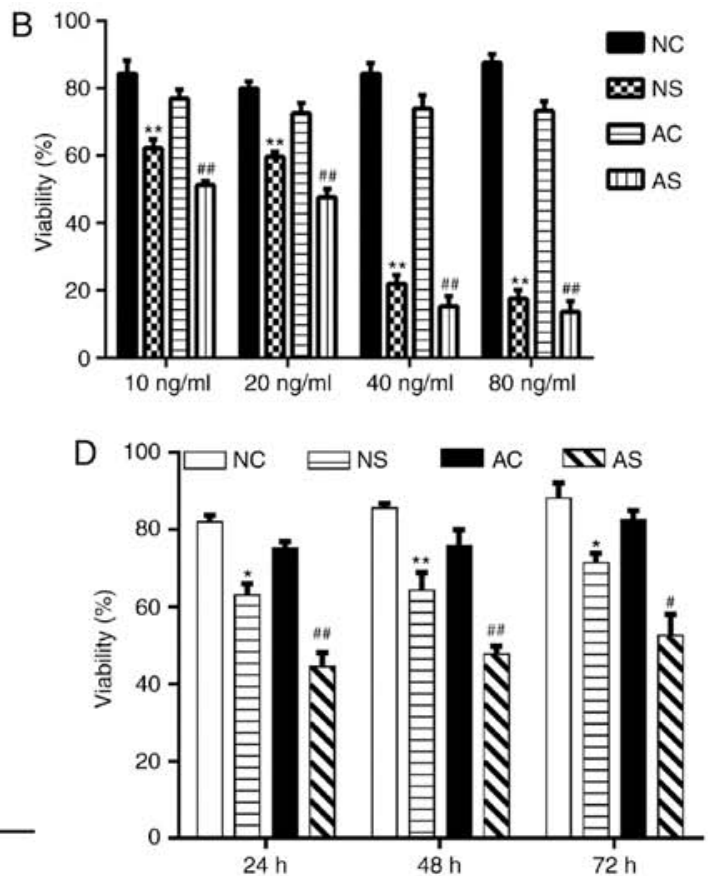

$48 \mathrm{~h}$
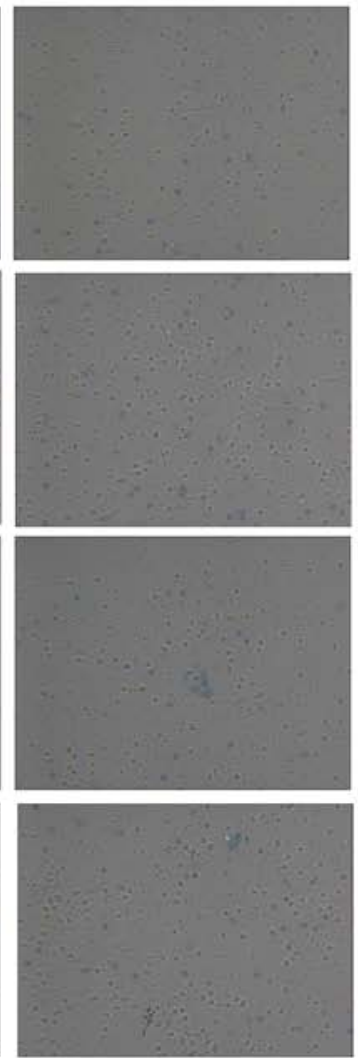
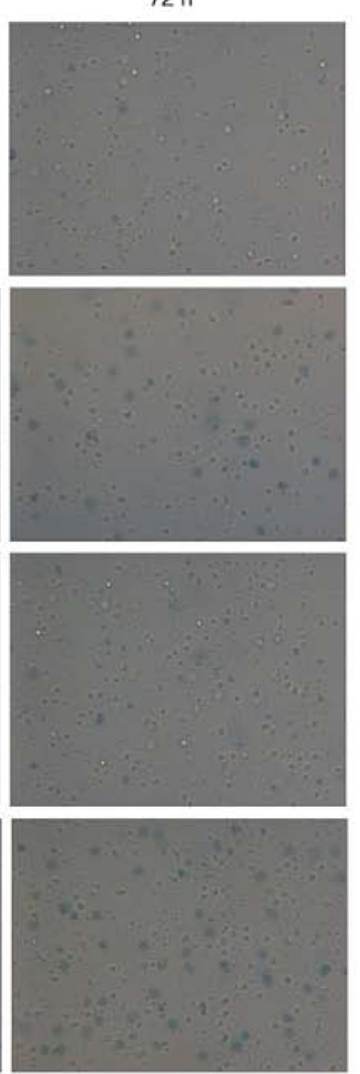

Figure 1. Effects of LPS on cell viability in monocytes from newborns and healthy adults. (A) The expression of VDR after treatment with LPS. (B) Percentage of trypan blue staining-negative cells in total cells (viability \%) upon treatment with different concentrations of LPS (10, 20,40 and $80 \mathrm{ng} / \mathrm{ml})$. (C) Monocytes of newborns and healthy adults were stimulated with or without LPS $(10 \mathrm{ng} / \mathrm{ml})$ at 24,48 and $72 \mathrm{~h}$, respectively. OD at $450 \mathrm{~nm}$ revealed the cell viability as determined by a Cell Counting Kit-8. (D and E) Percentage of trypan blue staining negative cells in total cells (viability \%) and representative images (magnification, $\mathrm{x} 200$ ). ${ }^{*} \mathrm{P}<0.05,{ }^{* *} \mathrm{P}<0.01$, NS vs. NC; ${ }^{~} \mathrm{P}<0.05,{ }^{\# \#} \mathrm{P}<0.01$, AS vs. AC. AC, adult control; AS, adult sepsis; NC: Newborn control; NS, newborn sepsis; LPS, lipopolysaccharide; OD, optical density; VDR, vitamin D receptor.

LPS (10 ng/ml) to induce a sepsis cell model. The viability of monocytes from the NC, NS, AC and AS groups was determined at 24,48 or $72 \mathrm{~h}$ after treatment by a CCK-8 assay and trypan blue assay, respectively. As presented in Fig. 1C, the
CCK-8 assay indicated that the viability of monocytes was significantly decreased in the NS and AS groups after $72 \mathrm{~h}$ of treatment compared with the corresponding controls. In the trypan blue assay, monocyte viability was significantly 

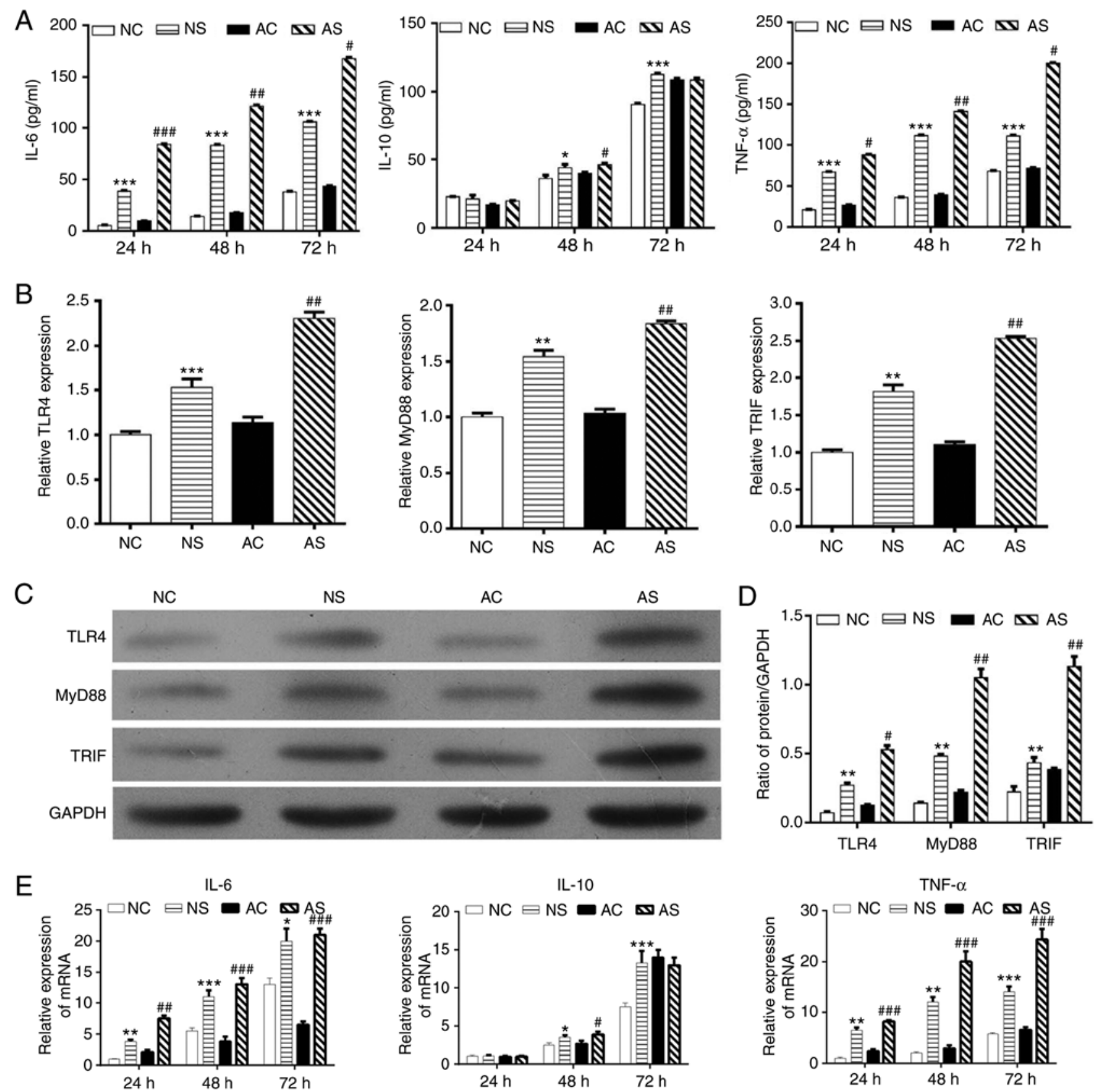

Figure 2. Effects of LPS stimulation on inflammation-related cytokines. (A) Levels of TNF- $\alpha$, IL-6 and IL-10 in the culture supernatants from monocytes in the NC, NS, AC, and AS groups at 24, 48 and $72 \mathrm{~h}$ by ELISA. (B) mRNA expression of TLR4, MyD88 and TRIF evaluated by RT-qPCR. (C) Representative blot of TLR4, MyD88 and TRIF from western blotting. (D) Densitometry analysis of TLR4, MyD88 and TRIF expression; monocytes of newborns and healthy adults were stimulated with or without LPS for $48 \mathrm{~h}$. (E) mRNA expression levels of IL-6, IL-10 and TNF- $\alpha$ in the culture supernatants from monocytes in the NC, NS, AC and AS groups at 24,48 and $72 \mathrm{~h}$ by $\mathrm{RT}-\mathrm{qPCR} .{ }^{*} \mathrm{P}<0.05,{ }^{* *} \mathrm{P}<0.01,{ }^{* * *} \mathrm{P}<0.001, \mathrm{NS}$ vs. NC; ${ }^{\#} \mathrm{P}<0.05$, ${ }^{\# \#} \mathrm{P}<0.01,{ }^{\# \# \#} \mathrm{P}<0.001, \mathrm{AS}$ vs. AC. AC, adult control; AS, adult sepsis; NC, newborn control; NS, newborn sepsis; IL, interleukin; LPS, lipopolysaccharide; MyD88, myeloid differentiation primary response gene 88 ; RT-qPCR, reverse transcription-quantitative PCR; TLR4, Toll-like receptor 4; TNF- $\alpha$, tumor necrosis factor- $\alpha$; TRIF, Toll-IL-1 resistance-domain-containing adapter-inducing interferon- $\beta$.

decreased in the NS and AS groups compared with the corresponding controls (Fig. 1D and E).

Subsequently, the effects of LPS on the release of cytokines were determined. As shown in Fig. 2A and E, IL-6 and TNF- $\alpha$ production in monocytes from newborns and healthy adults with sepsis was significantly increased with LPS treatment compared with the respective controls. Anti-inflammatory cytokine IL-10 expression was increased with LPS treatment for $48 \mathrm{~h}$ in the NS and AS groups compared with the controls; a significantly higher IL-10 level was observed in the NS group compared with NC group at $72 \mathrm{~h}$. However, no significant changes were observed after $72 \mathrm{~h}$ of LPS stimulation in monocytes from healthy adults. We also investigated whether LPS stimulation regulated TLR signaling. As shown in Fig. 2B, the expression levels of TLR4, MyD88 and TRIF mRNA in monocytes from the NS and AS groups were significantly increased when compared with the NC or AC groups. These results were further confirmed by western blotting (Fig. 2C and D). Therefore, LPS stimulation for $48 \mathrm{~h}$ was used in subsequent experiments. 

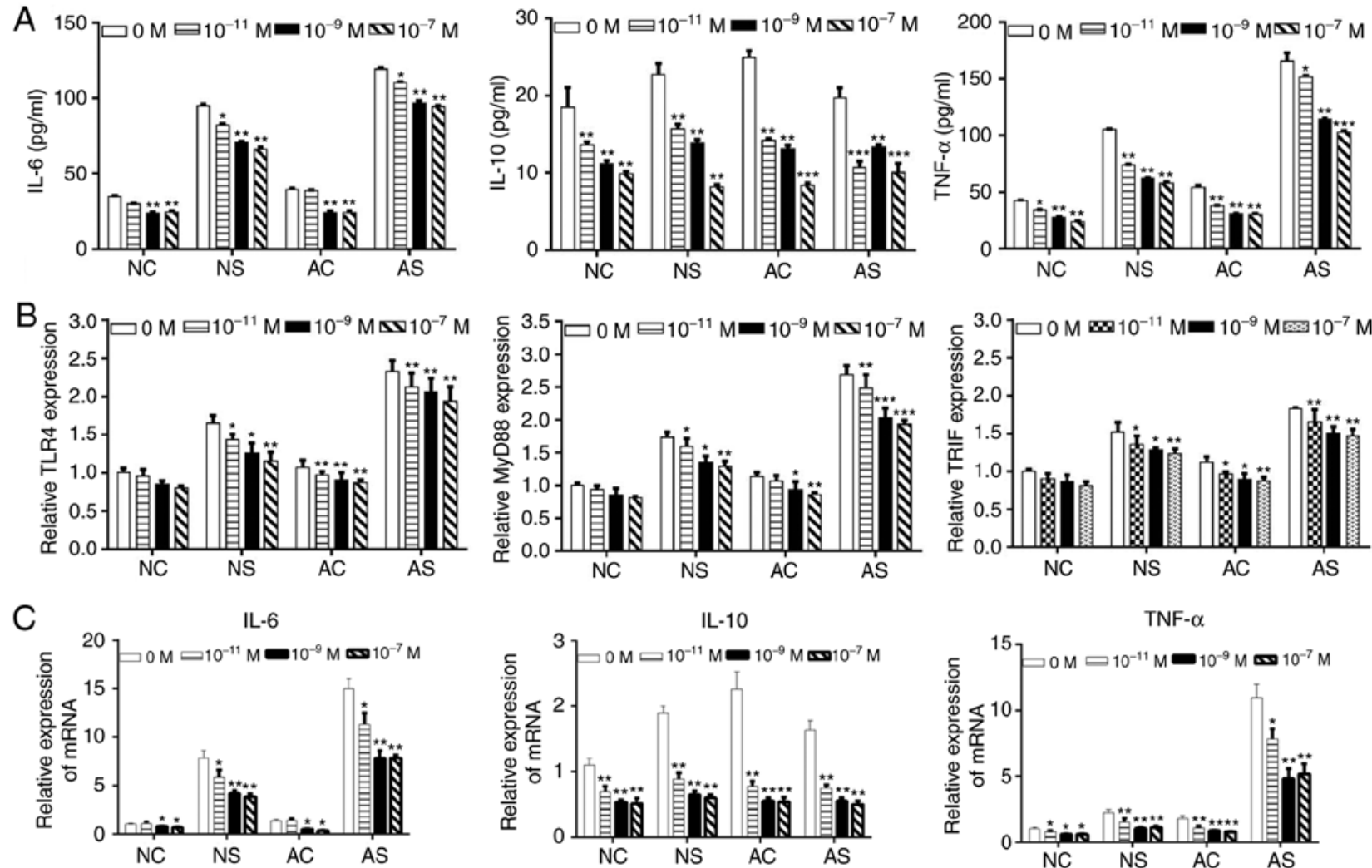

Figure 3. Effects of VD on IL-6, IL-10 and TNF- $\alpha$ release, and TLR signaling in lipopolysaccharide-stimulated monocytes from newborns and healthy adults. (A) Levels of IL-6, IL-10 and TNF- $\alpha$ in the NC, NS, AC and AS groups after treatment with VD at concentrations of $10^{-7}, 10^{-9}$ and $10^{-11} \mathrm{M}$ by ELISA. (B) TLR4, MyD88 and TRIF mRNA expression in the NC, NS, AC and AS groups after treatment with VD at concentrations of $10^{-7}, 10^{-9}$ and $10^{-11} \mathrm{M}$. (C) mRNA expression levels of IL-6, IL-10 and TNF- $\alpha$ in the NC, NS, AC and AS groups after treatment with VD at concentrations of $10^{-7}, 10^{-9}$ and $10^{-11} \mathrm{M}$ by reverse transcription-quantitative PCR. ${ }^{*} \mathrm{P}<0.05,{ }^{* *} \mathrm{P}<0.01,{ }^{* * *} \mathrm{P}<0.001$ vs. $0 \mathrm{M}$ VD. AC, adult control; AS, adult sepsis; NC, newborn control; NS, newborn sepsis; VD, 1,25-dihydroxy vitamin D3; IL, interleukin; MyD88, myeloid differentiation primary response gene 88; TLR4, Toll-like receptor 4; TNF- $\alpha$, tumor necrosis factor- $\alpha$; TRIF, Toll-IL-1 resistance-domain-containing adapter-inducing interferon- $\beta$.

VD attenuates LPS-induced pro-inflammatory cytokines release in monocytes. VD has been reported to have anti-inflammatory and immune regulatory functions $(34,35)$. We investigated whether VD could attenuate LPS-induced cytokine release and TLR4 signaling in monocytes from newborns and healthy adults. Monocytes were treated with VD $\left(10^{-7}, 10^{-9}\right.$ or $\left.10^{-11} \mathrm{M}\right)$ and LPS for $48 \mathrm{~h}$. As presented in Fig. 3A and $\mathrm{C}$, the expression of IL-6, IL-10 and TNF- $\alpha$ decreased in the NC, NS, AC and AS groups in a VD concentration-dependent manner. RT-qPCR indicated that the expression of TLR4, MyD88 and TRIF decreased following VD treatment in the four monocyte groups (Fig. 3B).

Anti-inflammatory effects of VD in LPS-stimulated monocytes is mediated by TLR4 signaling. To further investigate whether the anti-inflammatory effects of VD in monocytes occur in a TLR4-dependent manner, anti-TLR4 antibody was used to inhibit TLR4 signaling. An IgG antibody was used as isotype control. In the NC, NS, AC and AS groups, the cells were treated with IgG or anti-TLR4, IgG + VD or anti-TLR4 + VD. The levels of IL-6, IL-10 and TNF- $\alpha$ were evaluated by ELISA. As shown in Fig. 4A and C, blockade of TLR4 significantly downregulated the levels of IL- 6 and TNF- $\alpha$ but not IL-10. Furthermore, inhibition of TLR4 significantly decreased the levels of MyD88 and TRIF mRNA compared with the IgG group, as detected by RT-qPCR (Fig. 4B).
We also used si-RNA targeting MyD88 (siMyD88) and TRIF (siTRIF) to further confirm our findings (Fig. S1). As shown in Figs. 5A and C, and 6A, downregulation of MyD88 or TRIF significantly suppressed IL- 6 and TNF- $\alpha$ production in the siMyD88 or siTRIF, and siMyD88 + VD or siTRIF + VD groups compared with the siCon and siCon + VD groups, respectively. On the contrary, no significant difference was observed in IL-10 levels in these groups. siMyD88 and siTRIF treatment significantly decreased the expression of TLR4, MyD88 and TRIF (Figs. 5B and 6B) in monocytes from siMyD88 or siTRIF, and siMyD88 + VD or siTRIF + VD groups compared with the siCon and siCon + VD groups, respectively.

\section{Discussion}

The results of the present study revealed that LPS stimulation significantly attenuated cell viability, increased the production of pro-inflammatory cytokines (IL- 6 and TNF- $\alpha$ ), and promoted the expression of TLR4, MyD88 and TRIF. VD was determined to inhibit pro-inflammatory responses in monocytes induced by LPS stimulation involving the TLR4/MyD88-dependent and TRIF-dependent signaling pathways. Deficiency of TLR4, MyD88 or TRIF impaired the expression of the remaining two, which was accompanied with low levels of IL- 6 and TNF- $\alpha$. Thus, a feedback loop may occur, whereby the secretion of these pro-inflammatory cytokines is regulated. 
A

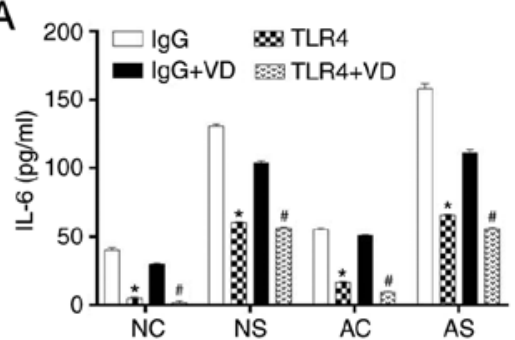

B
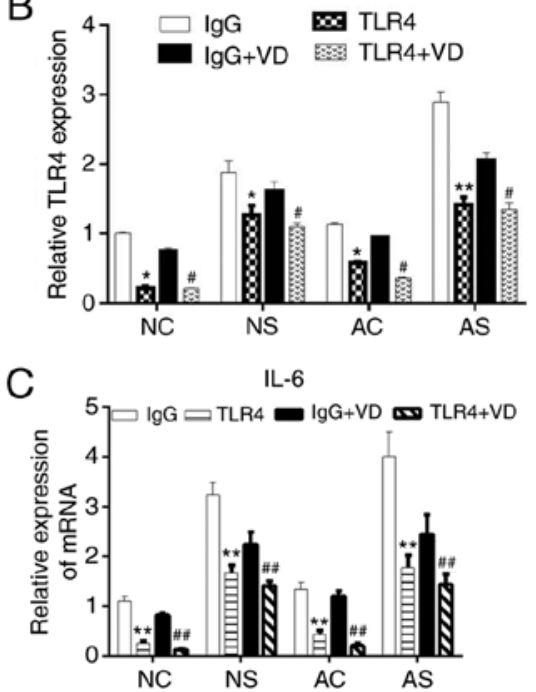
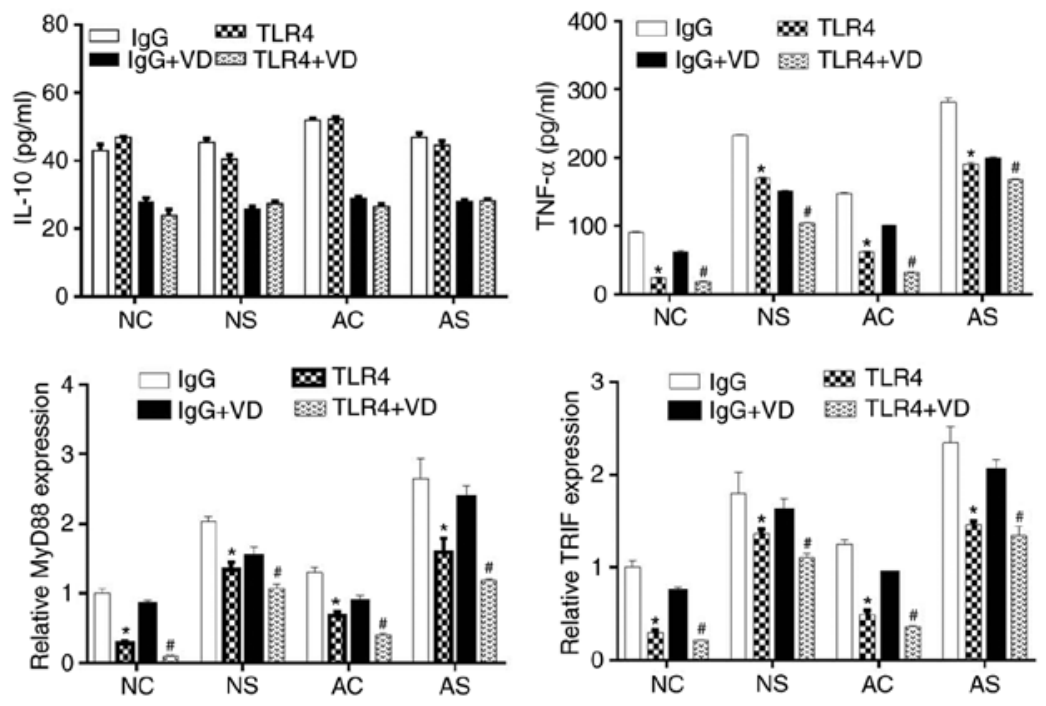

IL-10
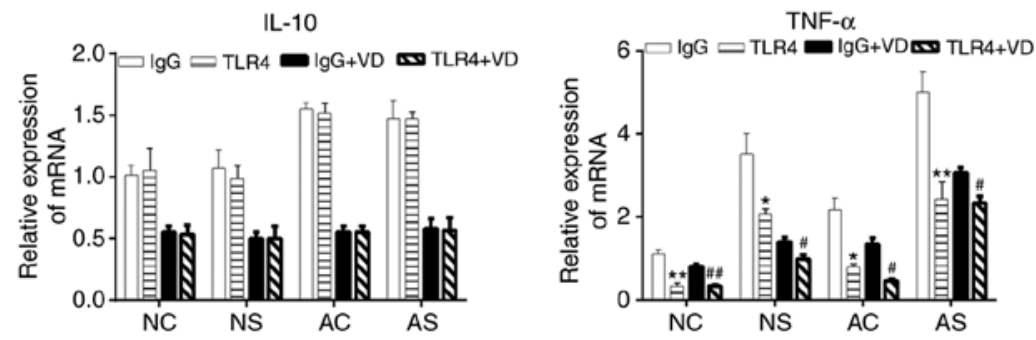

Figure 4. Effects of blocking TLR4 on the protein levels of TNF- $\alpha$, IL-6, IL-10, and mRNA levels of TLR4, MyD88 and TRIF in monocytes stimulated with lipopolysaccharide. (A) Levels of IL-6, IL-10 and TNF- $\alpha$ in the NC, NS, AC, and AS groups. The release of inflammation-related cytokines TNF- $\alpha$, IL-6, and IL-10 was measured in the culture supernatants from monocytes in four groups (NC, NS, AC and AS) after treatment with VD at concentration of $10^{-9} \mathrm{M}^{2}$ by ELISA. (B) mRNA levels of TLR4, MyD88 and TRIF in the four groups (NC, NS, AC and AS) after treatment with VD at concentration of $10^{-9}$. (C) Levels of IL-6, IL-10 and TNF- $\alpha$ mRNA in the culture supernatants from monocytes in the four groups (NC, NS, AC and AS) after treatment with VD at a concentration of $10^{-9} \mathrm{M}$ by reverse transcription-quantitative PCR. ${ }^{*} \mathrm{P}<0.05,{ }^{* *} \mathrm{P}<0.01$, IgG vs. TLR4; ${ }^{*} \mathrm{P}<0.05,{ }^{\# \#} \mathrm{P}<0.01$, IgG + VD vs. TLR4 + VD. AC, adult control; AS, adult sepsis; NC, newborn control; NS, newborn sepsis; VD, 1,25-dihydroxy vitamin D3; IL, interleukin; MyD88, myeloid differentiation primary response gene 88; TLR4, Toll-like receptor 4; TNF- $\alpha$, tumor necrosis factor- $\alpha$; TRIF, Toll-IL-1 resistance-domain-containing adapter-inducing interferon- $\beta$.

Cytokines are endogenous inflammatory proteins and function as mediators in the pathology and physiology of systemic inflammatory response syndrome (SIRS) caused by sepsis (36). Pro-inflammatory cytokines, such as TNF- $\alpha$, IL- 6 and IL- $\beta$ are essential for initiating the inflammatory process in host defense against infection, whereas overproduction of these cytokines contributes to multiple organ failure and mortality due to septic shock (37,38). Chawla et al (39) reported that a high concentration of IL- 6 acts as a risk factor for acute kidney injury in patients with severe sepsis. TNF- $\alpha$ appears to be a key cytokine in the pathogenesis of septic shock (40). In addition, anti-inflammatory factors, including transforming growth factor- $\beta$, IL-10 and prostaglandin E2 may be formed to alleviate persistent inflammation (41). Monocytes serve a critical role in the development of SIRS caused by sepsis (21). Activated monocytes in sepsis induce a dysfunctional immune-inflammatory response in which the production of anti-inflammatory cytokines, IL- 6 and TNF- $\alpha$ are released $(23,24)$. In the present study, significantly elevated expression levels of the pro-inflammatory cytokines IL- 6 and TNF- $\alpha$ were observed; however, the levels of the anti-inflammatory cytokine IL-10 were unaffected in monocytes treated with LPS. As the immune system of newborns is yet to develop further, we isolated monocytes from both newborns and healthy adults. Although a similar trend was observed compared between the treatment group and control group, the levels of inflammatory factors in newborns were generally lower than those in healthy adults (42). These findings supported the fact that monocytes serve an important role in the development of sepsis.

Emerging evidence from clinical studies has revealed a high prevalence of VD deficiency or insufficiency among patients with severe infections, such as sepsis (9). A previous study has proposed that VD had a modulatory effect on the inflammatory response in animal models of sepsis (43). In the present study, VD reduced the expression of pro-inflammatory factors (IL-6 and TNF- $\alpha$ ) in a dose-dependent manner, suggesting that VD exerted an inhibitory effect on inflammatory reactions; thus, VD may reduce sepsis-induced organ dysfunction and mortality.

MyD88 was identified as a necessary component for the activation of all TLR-mediated innate immunity processes, and TRIF is involved in the TLR4-mediated MyD88-independent signaling pathway (44). To investigate the molecular mechanism underlying the suppression of inflammatory cytokine secretion by VD, we inhibited TLR4 and its downstream molecules. In this study, it was observed that blocking TLR4 in newborn and adult monocytes hindered IL- 6 and TNF- $\alpha$ production, and inhibited the expression of MyD88 and TRIF. VD treatment resulted in a dose-dependent decrease 

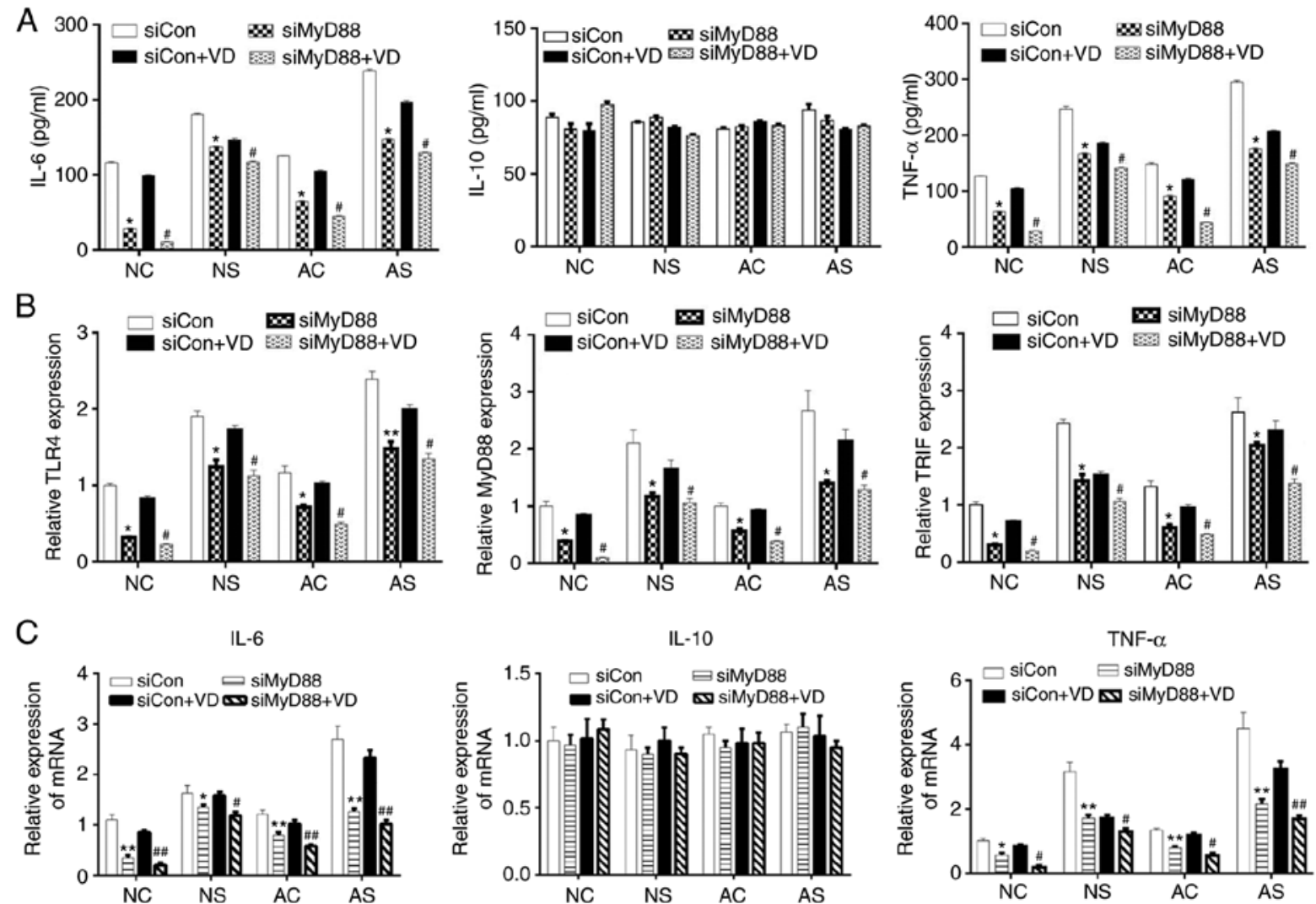

Figure 5. Effects of MyD88 downregulation on the expression of protein levels. TNF- $\alpha$, IL-6, IL-10, and mRNA levels of TLR4, TLR4 and TRIF. (A) The release of IL-6, IL-10 and TNF- $\alpha$ was measured by ELISA. (B) mRNA expression levels of TLR4, MyD88 and TRIF. (C) Levels of IL-6, IL-10 and TNF- $\alpha$ mRNA detected by reverse transcription-quantitative PCR. ${ }^{*} \mathrm{P}<0.05,{ }^{* *} \mathrm{P}<0.01$, siCon vs. siMyD88; ${ }^{*} \mathrm{P}<0.05,{ }^{\# \#} \mathrm{P}<0.01$, siCon + VD vs. siMyD88 + VD. AC, adult control; AS, adult sepsis; NC, newborn control; NS, newborn sepsis; VD, 1,25-dihydroxy vitamin D3; Con, control; IL, interleukin; MyD88, myeloid differentiation primary response gene 88 ; si, small interfering RNA; TLR4, Toll-like receptor 4; TNF- $\alpha$, tumor necrosis factor- $\alpha$; TRIF, Toll-IL-1 resistance-domain-containing adapter-inducing interferon- $\beta$.
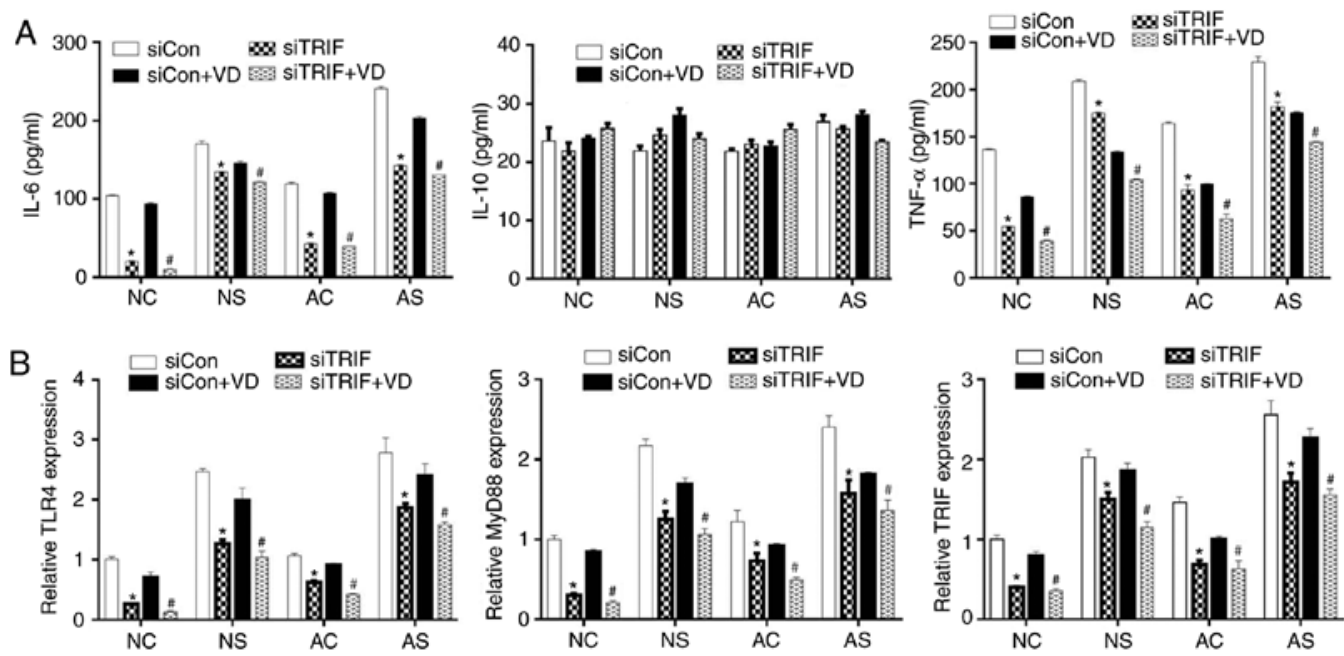

Figure 6. Effects of TRIF downregulation on the expression of TNF- $\alpha$, IL-6, IL-10, TLR4 and MyD88. (A) The release of IL-6, IL-10 and TNF- $\alpha$ was measured by ELISA. (B) mRNA expression levels of TLR4, MyD88 and TRIF as determined by reverse transcription-quantitative PCR. ${ }^{*} \mathrm{P}<0.05$, siCon vs. siTRIF; " $\mathrm{P}<0.05$, siCon + VD vs. siTRIF + VD. AC, adult control; AS, adult sepsis; NC, newborn control; NS, newborn sepsis; VD, 1,25-dihydroxy vitamin D3; Con, control; IL, interleukin; MyD88, myeloid differentiation primary response gene 88; si, small interfering RNA; TLR4, Toll-like receptor 4; TNF- $\alpha$, tumor necrosis factor- $\alpha$; TRIF, Toll-IL-1 resistance-domain-containing adapter-inducing interferon- $\beta$.

in TLR4 expression and its downstream molecules, MyD88 and TRIF. Evidence has shown that TLR-4 mRNA was downregulated after treatment with anti-TLR4 antibody (45). Anti-TLR4 monoclonal antibody attenuated lung injury caused by mechanical ventilation, inflammation and edema in rats by inhibiting the TLR4/MyD88 signaling pathway (46). Consistently, we observed that TLR4 was also decreased after treatment with the anti-TLR4 antibody, suggesting that 
the inhibition of TLR4 interferes with the cascades involving the TLR4/MyD88 signaling pathway. To the best of our knowledge, our study is the first to demonstrate for the first time that VD induced the suppression of IL- 6 and TNF- $\alpha$ in sepsis, which is likely mediated by the inhibition of the TLR4-MyD88-dependent and TRIF-dependent pathways. Of note, VD did not significantly affect the production of IL-10 in LPS-stimulated human monocytes.

Feedback mechanisms are necessary to sustain effective immunity, inflammation and tissue homeostasis (47). Hegyi et al (48) demonstrated a disturbance of AMP in psoriasis mediated by a pro-inflammatory feedback loop was induced by the VD analog, calcipotriol. White (49) confirmed that the VD-signaling pathway can be induced though TLR2 or TLR4 signaling via elevated VDR and VS 1- $\alpha$-hydroxylase expression; a positive feedback loop is formed with the involvement of VD, TLR2 and CD14. Interestingly, downregulation of MyD88 suppressed the pro-inflammatory response, and reduced the levels of TLR4 and TRIF. A similar pattern of results was observed in the cells with TRIF silencing. Thus, we proposed the hypothesis that the effects of VD on TLR4, MyD88 and TRIF expression in monocytes constituted a feedback loop in sepsis, which may be associated with the biosynthesis of pro-inflammatory cytokines. Our preliminary data suggest the role of VD in the attenuation of inflammation induced by LPS. However, the effects of VD in the treatment of sepsis should be further evaluated within in vivo models, such as LPS induction and cecal ligation models.

In summary, the present study reported that VD decreased the release of the pro-inflammatory cytokines IL- 6 and TNF- $\alpha$ by inhibiting the TLR4/MyD88 and TLR4/TRIF signaling pathways in LPS-stimulated monocytes. Our findings suggest that VD may be considered as a potential therapeutic agent in the treatment of sepsis, given its reported anti-inflammatory properties.

\section{Acknowledgements}

Not applicable.

\section{Funding}

This study was supported by Wenzhou Science \& Technology Bureau Project (grant. nos. Y20150128 and Y20180236), Wenzhou Health Bureau Project (grant. no. 2015B37), and Ruian Science \& Technology Bureau Project (grant. no. MS2017006).

\section{Availability of data and materials}

The analyzed datasets generated during the study are available from the corresponding author on reasonable request.

\section{Authors' contributions}

GZ and ZL conceived and designed the experiments. GZ, $\mathrm{NW}, \mathrm{YH}$, and MP performed the experiments. GZ analyzed the data. GZ and ZL wrote the paper. All authors read and approved the final manuscript.

\section{Ethics approval and consent to participate}

The research protocols were approved by the Ethics committee of Ruian People's Hospital. Written informed consent was provided by patients and/the patient's family.

\section{Patient consent for publication}

Not applicable.

\section{Competing interests}

The authors declare that they have no competing interests.

\section{References}

1. Rivers E, Nguyen B, Havstad S, Ressler J, Muzzin A, Knoblich B, Peterson E, Tomlanovich M and Early Goal-Directed Therapy Collaborative Group: Early goal-directed therapy in the treatment of severe sepsis and septic shock. N Engl J Med 345: 1368-1377, 2001.

2. Waydhas C, Nast-Kolb D, Jochum M, Trupka A, Lenk S, Fritz H, Duswald KH and Schweiberer L: Inflammatory mediators, infection, sepsis, and multiple organ failure after severe trauma. Arch Surg 127: 460-467, 1992.

3. Barber RC, Chang LY, Arnoldo BD, Purdue GF, Hunt JL, Horton JW and Aragaki CC: Innate immunity SNPs are associated with risk for severe sepsis after burn injury. Clin Med Res 4: 250-255, 2006

4. Aird WC: The role of the endothelium in severe sepsis and multiple organ dysfunction syndrome. Blood 101: 3765-3777, 2003.

5. Bar-Or D, Carrick MM, Mains CW, Rael LT, Slone D and Brody EN: Sepsis, oxidative stress, and hypoxia: Are there clues to better treatment? Redox Rep 20: 193-197, 2015.

6. Kaukonen KM, Bailey M, Suzuki S, Pilcher D and Bellomo R: Mortality related to severe sepsis and septic shock among critically ill patients in Australia and New Zealand, 2000-2012. JAMA 311: 1308-1316, 2014.

7. Holick MF: Vitamin D deficiency. N Engl J Med 357: 266-281, 2007.

8. Ginde AA, Camargo CA Jr and Shapiro NI: Vitamin D insufficiency and sepsis severity in emergency department patients with suspected infection. Acad Emerg Med 18: 551-554, 2011.

9. Jeng L, Yamshchikov AV, Judd SE, Blumberg HM, Martin GS, Ziegler TR and Tangpricha V: Alterations in vitamin D status and anti-microbial peptide levels in patients in the intensive care unit with sepsis. J Transl Med 7: 28, 2009.

10. Cecchi A, Bonizzoli M, Douar S, Mangini M, Paladini S, Gazzini B, Degl'Innocenti S, Linden M, Zagli G and Peris A: Vitamin D deficiency in septic patients at ICU admission is not a mortality predictor. Minerva Anestesiol 77: 1184-1189, 2011.

11. Moller S, Laigaard F, Olgaard K and Hemmingsen C: Effect of 1,25-dihydroxy-vitamin D3 in experimental sepsis. Int J Med Sci 4: 190-195, 2007.

12. Hewison M, Zehnder D, Chakraverty R and Adams JS: Vitamin D and barrier function: A novel role for extra-renal 1 alpha-hydroxylase. Mol Cell Endocrinol 215: 31-38, 2004.

13. Matsumoto M, Kikkawa S, Kohase M, Miyake K and Seya T: Establishment of a monoclonal antibody against human Toll-like receptor 3 that blocks double-stranded RNA-mediated signaling. Biochem Biophys Res Commun 293: 1364-1369, 2002.

14. Kawasaki $\mathrm{T}$ and Kawai T: Toll-like receptor signaling pathways. Front Immunol 5: 461, 2014.

15. Akira S and Takeda K: Toll-like receptor signalling. Nat Rev Immunol 4: 499-511, 2004.

16. Rathinam VA, Appledorn DM, Hoag KA, Amalfitano A and Mansfield LS: Campylobacter jejuni-induced activation of dendritic cells involves cooperative signaling through Toll-like receptor 4 (TLR4)-MyD88 and TLR4-TRIF axes. Infect Immun 77: 2499-2507, 2009.

17. Medzhitov R, Preston-Hurlburt P, Kopp E, Stadlen A, Chen C, Ghosh S and Janeway CA Jr: MyD88 is an adaptor protein in the hToll/IL-1 receptor family signaling pathways. Mol Cell 2: 253-258, 1998. 
18. Yamamoto $M$ and Akira S: TIR domain-containing adaptors regulate TLR-mediated signaling pathways. Nihon Rinsho 62: 2197-2203, 2004 (In Japanese).

19. Yamamoto M, Sato S, Hemmi H, Hoshino K, Kaisho T, Sanjo H, Takeuchi O, Sugiyama M, Okabe M, Takeda K and Akira S: Role of adaptor TRIF in the MyD88-independent toll-like receptor signaling pathway. Science 301: 640-643, 2003.

20. Horng T, Barton GM and Medzhitov R: TIRAP: An adapter molecule in the Toll signaling pathway. Nat Immunol 2: 835-841, 2001.

21. Shalova IN, Kajiji T, Lim JY, Gómez-Piña V, Fernández-Ruíz I, Arnalich F, Iau PT, López-Collazo E, Wong SC and Biswas SK: CD16 regulates TRIF-dependent TLR4 response in human monocytes and their subsets. J Immunol 188: 3584-3593, 2012.

22. Castanheira FVES, de Lima KA, Cebinelli GCM, Sônego F, Kanashiro A, Colon DF, Borges V, Czaikoski PG, Mota JM, Cunha TM, et al: CCR5-Positive inflammatory monocytes are crucial for control of sepsis. Shock Dec 7, 2018 (Epub ahead of print).

23. Medzhitov R, Preston-Hurlburt P and Janeway CA Jr: A human homologue of the Drosophila Toll protein signals activation of adaptive immunity. Nature 388: 394-397, 1997.

24. Akira S: Innate immunity to pathogens: Diversity in receptors for microbial recognition. Immunol Rev 227: 5-8, 2009.

25. Rosadini CV, Zanoni I, Odendall C, Green ER, Paczosa MK, Philip NH, Brodsky IE, Mecsas J and Kagan JC: A single bacterial immune evasion strategy dismantles both MyD88 and TRIF signaling pathways downstream of TLR4. Cell Host Microbe 18: 682-693, 2015.

26. Bjorkbacka H, Fitzgerald KA, Huet F, Li X, Gregory JA, Lee MA, Ordija CM, Dowley NE, Golenbock DT and Freeman MW: The induction of macrophage gene expression by LPS predominantly utilizes Myd88-independent signaling cascades. Physiol Genomics 19: 319-330, 2004

27. Kawai T, Adachi O, Ogawa T, Takeda $\mathrm{K}$ and Akira S: Unresponsiveness of MyD88-deficient mice to endotoxin. Immunity 11: 115-122, 1999.

28. Diomede F, Zingariello M, Cavalcanti MFXB, Merciaro I, Pizzicannella J, De Isla N, Caputi S, Ballerini P and Trubiani O: MyD88/ERK/NFkB pathways and pro-inflammatory cytokines release in periodontal ligament stem cells stimulated by Porphyromonas gingivalis. Eur J Histochem 61: 2791, 2017.

29. Cheong C, Matos I, Choi JH, Dandamudi DB, Shrestha E, Longhi MP, Jeffrey KL, Anthony RM, Kluger C, Nchinda G, et al: Microbial stimulation fully differentiates monocytes to DC-SIGN/CD209(+) dendritic cells for immune T cell areas. Cell 143: 416-429, 2010.

30. Murillo G, Nagpal V, Tiwari N, Benya RV and Mehta RG: Actions of vitamin D are mediated by the TLR4 pathway in inflammation-induced colon cancer. J Steroid Biochem Mol Biol 121: 403-407, 2010.

31. Sadeghi K, Wessner B, Laggner U, Ploder M, Tamandl D, Friedl J, Zügel U, Steinmeyer A, Pollak A, Roth E, et al: Vitamin D3 down-regulates monocyte TLR expression and triggers hyporesponsiveness to pathogen-associated molecular patterns. Eur J Immunol 36: 361-370, 2006.

32. Livak KJ and Schmittgen TD: Analysis of relative gene expression data using real-time quantitative PCR and the 2(-Delta Delta C(T)) method. Methods 25: 402-408, 2001.

33. Giannoni E, Guignard L, Knaup Reymond M, Perreau M, Roth-Kleiner M, Calandra T and Roger T: Estradiol and progesterone strongly inhibit the innate immune response of mononuclear cells in newborns. Infect Immun 79: 2690-2698, 2011.
34. Manion M, Hullsiek KH, Wilson EMP, Rhame F, Kojic E, Gibson D, Hammer J, Patel P, Brooks JT, Baker JV, et al: Vitamin D deficiency is associated with IL-6 levels and monocyte activation in HIV-infected persons. PLoS One 12: e0175517, 2017.

35. Wei R and Christakos S: Mechanisms underlying the regulation of innate and adaptive immunity by vitamin D. Nutrients 7: 8251-8260, 2015.

36. Gogos CA, Drosou E, Bassaris HP and Skoutelis A: Pro- versus anti-inflammatory cytokine profile in patients with severe sepsis: A marker for prognosis and future therapeutic options. J Infect Dis 181: 176-180, 2000.

37. Pinsky MR, Vincent JL, Deviere J, Alegre M, Kahn RJ and Dupont E: Serum cytokine levels in human septic shock. Relation to multiple-system organ failure and mortality. Chest 103: 565-575, 1993.

38. Parrillo JE, Parker MM, Natanson C, Suffredini AF, Danner RL, Cunnion RE and Ognibene FP: Septic shock in humans. Advances in the understanding of pathogenesis, cardiovascular dysfunction, and therapy. Ann Intern Med 113: 227-242, 1990.

39. Chawla LS, Seneff MG, Nelson DR, Williams M, Levy H, Kimmel PL and Macias WL: Elevated plasma concentrations of IL-6 and elevated APACHE II score predict acute kidney injury in patients with severe sepsis. Clin J Am Soc Nephrol 2: 22-30, 2007.

40. Mira JP, Cariou A, Grall F, Delclaux C, Losser MR, Heshmati F, Cheval C, Monchi M, Teboul JL, Riché F, et al: Association of TNF2, a TNF-alpha promoter polymorphism, with septic shock susceptibility and mortality: A multicenter study. JAMA 282: 561-568, 1999.

41. Haveman JW, Muller Kobold AC, Tervaert JW, van den Berg AP, Tulleken JE, Kallenberg CG and The TH: The central role of monocytes in the pathogenesis of sepsis: Consequences for immunomonitoring and treatment. Neth J Med 55: 132-141, 1999.

42. Li YP, Yu SL, Huang ZJ, Huang J, Pan J, Feng X, Zhang XG, Wang $\mathrm{JH}$ and Wang J: An impaired inflammatory cytokine response to gram-negative LPS in human neonates is associated with the defective TLR-mediated signaling pathway. J Clin Immunol 35: 218-226, 2015.

43. Watkins RR, Yamshchikov AV, Lemonovich TL and Salata RA: The role of vitamin D deficiency in sepsis and potential therapeutic implications. J Infect 63: 321-326, 2011.

44. Takeda K and Akira S: TLR signaling pathways. Semin Immunol 16: 3-9, 2004.

45. Zhou S, Wang G and Zhang W: Effect of TLR4/MyD88 signaling pathway on sepsis-associated acute respiratory distress syndrome in rats, via regulation of macrophage activation and inflammatory response. Exp Ther Med 15: 3376-3384, 2018.

46. Huang C, Pan L, Lin F, Dai H and Fu R: Monoclonal antibody against Toll-like receptor 4 attenuates ventilator-induced lung injury in rats by inhibiting MyD88- and NF-kappaB-dependent signaling. International Int J Mol Med 39: 693-700, 2017.

47. Chen Y, Liu W, Sun T, Huang Y, Wang Y, Deb DK, Yoon D, Kong J, Thadhani R and Li YC: 1,25-Dihydroxyvitamin D promotes negative feedback regulation of TLR signaling via targeting microRNA-155-SOCS1 in macrophages. J Immunol 190: 3687-3695, 2013.

48. HegyiZ,ZwickerS, BureikD,Peric M,Koglin S, Batycka-Baran A, Prinz JC, Ruzicka T, Schauber J and Wolf R: Vitamin D analog calcipotriol suppresses the Th17 cytokine-induced proinflammatory S100 'alarmins' psoriasin (S100A7) and koebnerisin (S100A15) in psoriasis. J Invest Dermatol 132: 1416-1424, 2012.

49. White JH: Vitamin D signaling, infectious diseases, and regulation of innate immunity. Infect Immun 76: 3837-3843, 2008. 